\title{
Correção de sorriso gengival associada à frenotomia labial superior em paciente com Erupção Passiva Alterada (EPA): um relato de caso
}

\author{
Correction of gingival smile associated with upper lip frenotomy in a patient with Altered \\ Passive Eruption (EPA): a case report
}

Corrección de la sonrisa gingival asociada con la frenotomía del labio superior en un paciente con Erupción Pasiva Alterada (EPA): reporte de un caso

Quemuel Pereira da Silva1, Ioana Veras Silva Barreto², Paula Lima Nogueira1, Maria Vitoria Oliveira Dantas $^{1 *}$, Amanda da Silva Araújo ${ }^{1}$, Filipe de Oliveira Lima1, Júlia Tavares Palmeira ${ }^{1}$, Kadmo Azevedo de Figueiredo², Samara Cirilo Feitosa Germano², Ítalo Cardoso dos Santos ${ }^{3}$.

\section{RESUMO}

Objetivo: relatar o que é referente às possibilidades terapêuticas, diagnósticas e aos aspectos clínicos do procedimento de correção de sorriso gengival pela técnica de gengivectomia em bisel externo e reposicionamento de freio labial superior. Detalhamento do caso: o presente caso é referente ao paciente S.V.L.B.S, do sexo masculino, 17 anos, melanoderma, estudante, com queixa de "mostrar muita gengiva ao sorrir", diagnosticado com erupção passiva alterada (EPA) do tipo 1 e subtipo A. Sendo assim, o tratamento escolhido foi a intervenção periodontal pela técnica de gengivectomia em bisel externo e gengivoplastia da área compreendida entre os elementos 14 ao $24 \mathrm{com}$ posterior frenotomia labial superior. No presente caso clínico houve acompanhamento de 3 meses, e durante todo esse tempo o resultado e aparência clínica se mantiveram satisfatórios. Considerações finais: Diante disso a técnica de gengivectomia em bisel externo e plastia gengival no tratamento da EPA pode se mostrar uma ótima alternativa nos casos dessas modificações estéticas, podendo assim, devolver a harmonia estética do sorriso ao paciente.

Palavras-chave: Periodontia, Freio labial, Gengivectomia.

\begin{abstract}
Objective: to report or refer to the therapeutic possibilities, diagnoses and clinical aspects of the procedure for correcting gingival smile using the gingivectomy technique on the external bevel and repositioning of the upper lip. Case details: the present case refers to the patient SVLBS, male, 17 years old, melanoderma, student, with a complaint of "showing too much gum when smiling", diagnosed with altered passive eruption (EPA) of type 1 and subtype A. thus, the treatment chosen was periodontal intervention using the external bevel gingivectomy technique and gingivoplasty of the area between elements 14 and 24 with posterior upper lip frenotomy. In the present clinical case, there was a 3-month follow-up, and throughout this time or result and clinical appearance remained satisfactory. Final considerations: Given this, the technique of external bevel gingivectomy and gingival plasty in the treatment of EPA can show a great alternative in cases of aesthetic changes, thus being able to restore an aesthetic harmony of the smile to the patient.
\end{abstract}

Keywords: Periodontics, Labial frenum, Gingivectomy.

\footnotetext{
${ }^{1}$ Universidade Federal de Campina Grande (UFCG), Patos-PB. *E-mail: mvitoria.od@hotmail.com

2 Centro Universitário de Patos (UNIFip), Patos-PB

${ }^{3}$ Faculdades Integradas de Patos CG (FIP-CG), Campina Grande-PB
} 


\section{RESUMEN}

Objetivo: informar o referirse a las posibilidades terapéuticas, diagnósticos y aspectos clínicos del procedimiento para corregir la sonrisa gingival, utilizando la técnica de gingivectomía en el chaflán externo y el reposicionamiento del labio superior. Detalles del caso: el presente caso se refiere al paciente SVLBS, hombre, 17 años, melanoderma, estudiante, con una queja de "mostrar exceso de encías al sonreír", diagnosticado con erupción pasiva alterada (EPA) tipo 1 y subtipo A. Por lo tanto, el tratamiento elegido fue la intervención periodontal, utilizando la técnica de gingivectomía con chaflán externo y gingivaplastia del área entre los elementos 14 y 24 con frenotomía posterior del labio superior. En el presente caso clínico, hubo un seguimiento de tres meses y, durante este período, el resultado y el aspecto clínico se mantuvieron satisfactorios. Consideraciones finales: Dado esto, la técnica de gingivectomía con chaflán externo y plastia gingival en el tratamiento de EPA puede ser una gran alternativa en casos de cambios estéticos, y puede restaurar una armonía estética de la sonrisa para el paciente.

Palabras clave: Periodoncia, Frenillo labial, Gingivectomía.

\section{INTRODUÇÃO}

$\mathrm{Na}$ atualidade, tudo o que engloba a estética dental está sendo amplamente procurada pela sociedade, com isso, a demanda por procedimentos odontológicos que oferecem esses tipos de intervenção vem só aumentando, e a busca por novas técnicas e aprimoramento das já existentes é uma realidade dos cirurgiões dentistas envolvidos nesse ambiente (DUTRA MB, et al., 2018).

O sorriso tem poder de influência sobre a estética e as expressões faciais, sendo o tecido gengival de extrema importância nessa relação, assim, existem vários fatores que podem atrapalhar a harmonia estética de um sorriso, como: erupção passiva alterada, coroa clínica curta, hiperatividade do lábio superior ou redução da espessura do mesmo e o acentuado crescimento vertical da maxila (SEIXAS MR, et al., 2011).

Dessa forma, a técnica de gengivectomia tanto em sua forma convencional como minimamente invasiva, tem sido descrita em ampla escala como procedimento com resolubilidade nesses casos, devido à qualidade dos seus resultados (DUTRA MB, et al., 2018; CRISTÓVAN AVS, et al., 2020).

Muitas vezes, após o tratamento com gengivectomia, o posicionamento do freio labial pode ser alterado e se apresentar baixo devido ao tecido gengival removido, com isso pode-se optar por técnicas cirúrgicas como a frenectomia, que consiste na completa ou parcial remoção do freio, a frenotomia, que consiste apenas em um reposicionamento do freio (VIEIRA PR, et al.,2014; SILVA HL, et al., 2018).

Diante disso, o objetivo do presente estudo é detalhar e discutir um caso clínico no qual foi realizada uma intervenção cirúrgica com a finalidade de corrigir um sorriso gengival, a partir da técnica de gengivectomia convencional em bisel externo e reposicionamento do freio labial superior.

\section{DETALHAMENTO DO CASO}

Paciente S.V.L.B.S, do sexo masculino, 17 anos, melanoderma, estudante, compareceu a clínica escola de odontologia da pós graduação em Periodontia do Centro Universitário de Patos - UNIFIP, acompanhado de maior responsável, queixando-se de "mostrar muita gengiva ao sorrir". Na anamnese o paciente não relatou nenhum fato que pudesse interferir no tratamento, nenhuma alteração sistêmica que pudesse corroborar com a sua condição periodontal.

Em exame físico intra-oral, analisou-se que o paciente apresentava ausência de perda de inserção, lábio em posição normal, fenótipo periodontal espesso com grande faixa de mucosa ceratinizada e linha dos zênites gengivais simétrica, porém apresentava as coroas clínicas dos elementos anterossuperiores curtas (Figura 1).

REAS/EJCH | Vol.12(12) | e4156 | DOI: https://doi.org/10.25248/reas.e4156.2020 Página 2 de 8 
Inicialmente foi realizado um exame periodontal detalhado com auxílio de uma sonda milimetrada Carolina do Norte PC15 (Millenium Golgran ${ }^{\circledR}$ ) dos elementos dentários 14 ao 24, onde foram considerados os seguintes critérios: mucosa ceratinizada e profundidade de sondagem. Obtendo-se esses dados foi constatado que o paciente apresentava profundidade de sondagem em valores entre 3 e $4 \mathrm{~mm}$ e sangramento a sondagem ausente (Quadro 1). Também foram solicitados exames complementares, mais especificamente o exame radiográfico periapical de boca toda, e exames laboratoriais de coagulograma, hemograma e glicemia em jejum.

Figura 1 - Condição inicial do paciente.

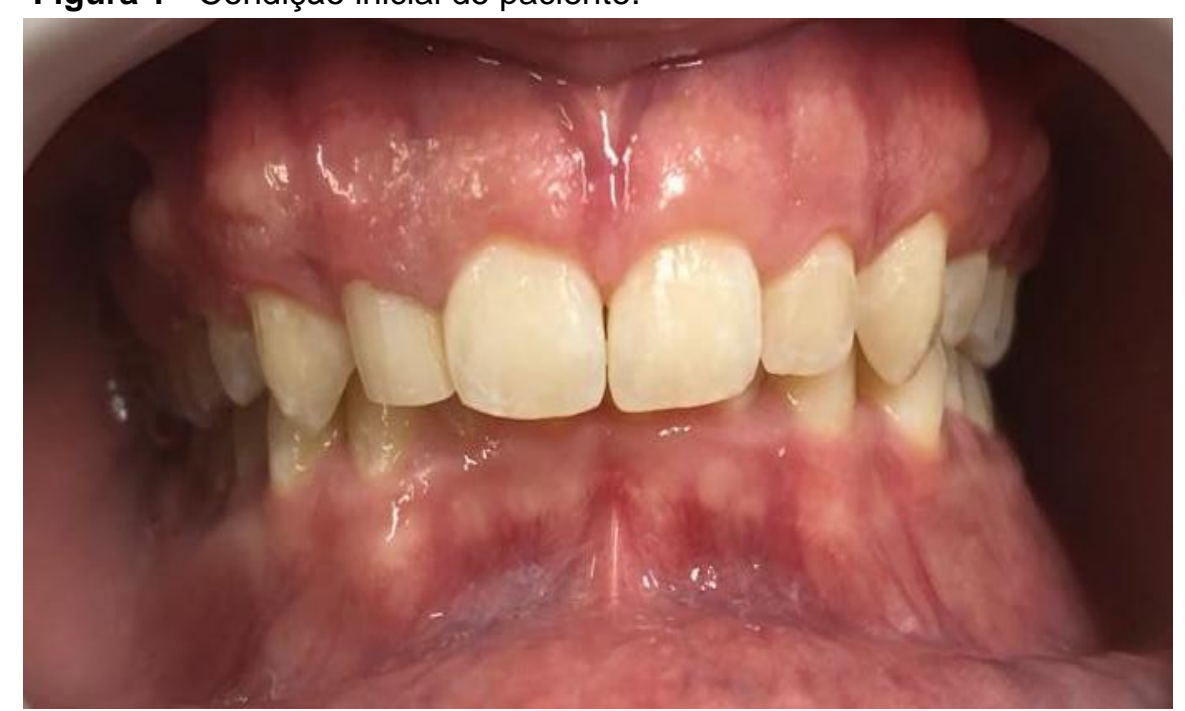

Fonte: Silva QP, et al., 2020.

Quadro 1 - Exame Periodontal detalhado.

\begin{tabular}{|c|c|c|c|c|c|c|c|c|}
\hline \multirow{2}{*}{ Dente } & \multicolumn{2}{|c|}{ MV } & \multicolumn{2}{|c|}{ V } & \multicolumn{2}{|c|}{ DV } & \multirow{2}{*}{$\begin{array}{l}\text { MC } \\
\text { MM }\end{array}$} & \multirow{2}{*}{$\begin{array}{c}\text { GI } \\
\text { MM } \\
\end{array}$} \\
\hline & $\mathbf{R}$ & $\mathbf{S}$ & $\mathbf{R}$ & $\mathbf{S}$ & $\mathbf{R}$ & $\mathbf{S}$ & & \\
\hline 14 & 0 & 3 & 0 & 3,5 & 0 & 3 & 10 & 6,5 \\
\hline 13 & 0 & 3,5 & 0 & 4 & 0 & 3,5 & 10 & 6 \\
\hline 12 & 0 & 3 & 0 & 3,5 & 0 & 3 & 9,5 & 6 \\
\hline 11 & 0 & 3,5 & 0 & 4 & 0 & 3,5 & 9 & 5 \\
\hline 21 & 0 & 3,5 & 0 & 4 & 0 & 3,5 & 9 & 5 \\
\hline 22 & 0 & 3 & 0 & 3,5 & 0 & 3 & 9,5 & 6 \\
\hline 23 & 0 & 3,5 & 0 & 4 & 0 & 3,5 & 10 & 6 \\
\hline 24 & 0 & 3 & 0 & 3,5 & 0 & 3 & 10 & 6,5 \\
\hline
\end{tabular}

Legenda: MV- face mesio-vestibular; V- face vestibular, DV- face disto-vestibular; MC- mucosa ceratinizada; GI- gengiva inserida; R- recessão; S-profundidade de sondagem; MM- milímetros. Fonte: Silva QP, et al., 2020. 
No encontro seguinte o paciente retornou com os exames em mãos. Não foram encontrados valores alterados em nenhum dos exames laboratoriais realizados. Nos exames radiográficos periapicais não foi observado perda óssea interproximal, confirmando assim que o paciente não apresentava perda de inserção clínica, indicando o diagnóstico de erupção passiva alterada (EPA) do tipo 1 e subtipo A, onde segundo Coslet, Vanarsdall e Weisgold (1977) o tipo 1 caracteriza-se pela presença de uma faixa mais larga de gengiva ceratinizada, desde a margem gengival até a linha mucogengival, que está situada apicalmente à crista óssea e mede mais de $2-3 \mathrm{~mm}$, e o subtipo $\mathrm{A}$ em que a distância desde a junção cemento-esmalte à crista óssea está dentro dos valores fisiológicos $(2,5$ a $3 \mathrm{~mm})$. A única alteração encontrada nos exames periapicais foi à anatomia conóide do elemento 12 que foi corrigida através de uma restauração em resina composta.

Com a hipótese diagnóstica, sucedeu-se a escolha do protocolo clínico, sendo escolhida a técnica de gengivectomia em bisel externo e Gengivoplastia da área compreendida entre os elementos 14 ao 24 . A técnica em bisel externo foi escolhida devido ao fato de não haver necessidade de confecção de retalho e osteotomia, visto que os tecidos supracrestais estavam estabelecidos corretamente, e a necessidade de uma plastia gengival foi observada para diminuir em espessura a mucosa ceratinizada do paciente. Já em relação à área abrangida pela intervenção, optou-se por ser realizada dos elementos 14 ao 24 devido ao sorriso expandido do paciente e a coroa clínica curta dos primeiros pré molares superiores.

Deu-se início então a intervenção cirúrgica, onde inicialmente foi realizada a antissepsia intra e extra-oral com digluconato de clorexidina a $0,12 \%$ e a $2 \%$, respectivamente. Em seguida o paciente foi anestesiado com auxílio de uma seringa carpule com agulha $30 \mathrm{G}$ e 2,5 tubetes de cloridato de mepivacaína a $2 \%$ com epinefrina 1:100.000, sendo realizadas as técnicas de bloqueio dos nervos alveolares superiores médio e anterior e infiltrativa papilar em toda a extensão da região a ser operada.

Após a anestesia sucedeu-se uma nova sondagem periodontal para se observar os tecidos supracrestais, ou seja, a distância entre a JCE e a crista óssea em todos os elementos, com isso, observou-se que os tecidos supracrestais estavam de fato dentro dos padrões de normalidade, variando entre 2 e $3 \mathrm{~mm}$. Tendo essas informações foi possível fechar o diagnóstico em EPA do tipo 1 e subtipo A.

Após isso, sucedeu-se a marcação da área de gengiva a ser removida com auxílio de uma sonda periodontal milimetrada Carolina do Norte $n^{\circ} 15$, sendo confeccionados pontos sangrantes para guiar a incisão nas alturas indicadas pela profundidade de sondagem de cada elemento levando em consideração também a altura e posição dos zênites gengivais de forma a garantir a harmonia estética (Quadro 1).

Em seguida foi realizada uma incisão paramarginal em bisel externo, onde o cabo de bisturi encontra-se voltado para a gengiva durante o corte, para isso foi usado um cabo de bisturi $\mathrm{N}^{\circ} 3$ (Golgran $\AA$ ) e 4 lâminas de bisturi do tipo 15c, uma para cada 2 dentes. Após essa primeira incisão, uma segunda do tipo intrasulcular foi realizada também com o auxílio dos mesmos instrumentais, para permitir um melhor desprendimento dos tecidos que se desejava remover. Em seguida com o auxílio de uma cureta periodontal Gracey 5-6 (Millenium Golgran $\circledast$ ) o colarinho gengival foi removido.

Após o tecido gengival em excesso ter sido removido, ainda com o auxílio de uma cureta periodontal Gracey 5-6, as superfícies dentárias agora expostas foram raspadas e alisadas removendo assim o tecido de granulação presente na área. Os cortes realizados com bisturi foram refinados com o auxílio de uma tesoura Castroviejo de $9 \mathrm{~cm}$ (Quinelato®), de forma a proporcionar um contorno mais adequado aos elementos dentários.

Posteriormente foi realizada a plastia gengival, com auxílio do gengivótomo de Kirkland (Millenium Golgran ${ }^{\circledR}$ ), para correção em espessura, coloração e textura gengival (Figura 2). Logo após, o cimento cirúrgico (Pericem - Technew $\left.{ }^{\circledR}\right)$ foi manipulado e colocado na ferida cirúrgica e as instruções para o período pós-operatório foram repassadas. Conduziu-se então a prescrição de Dipirona Sódica com ingestão por via oral de um comprimido de $500 \mathrm{mg}$ de seis em seis horas durante três dias e o lbuprofeno com ingestão por via oral de um comprimido de $600 \mathrm{mg}$ também de seis em seis horas durante cinco dias. Um novo encontro foi marcado inicialmente para 30 dias pós-intervenção, ou em caso de complicações pós-operatórias, em um intervalo de tempo menor. 
O paciente retornou após os 30 dias e não relatou nenhum problema durante o pós-operatório, apresentou uma boa cicatrização e resultado estético satisfatório, porém o mesmo ainda se queixou da posição de inserção do freio labial, que era visível quando sorria (Figura 3).

Com isso sucedeu-se com a realização de uma intervenção cirúrgica que consistiu no reposicionamento do freio labial para uma região mais apical com técnica semelhante à proposta por Bruder et al (2015), com a finalidade de manter as características estéticas e anatômicas do freio, visto que não foi feita sua excisão por completo. $O$ procedimento iniciou-se com a anestesia realizada com auxílio de seringa carpule, agulha 30G e 1 tubete de cloridato de mepivacaína a $\%$ com epinefrina 1:100.000, pela técnica infiltrativa local em fundo de sulco nas áreas adjacentes ao freio labial.

Figura 2 - Pós-operatório imediato do paciente, com visualização do tecido conjuntivo sangrante exposto após plastia gengival.

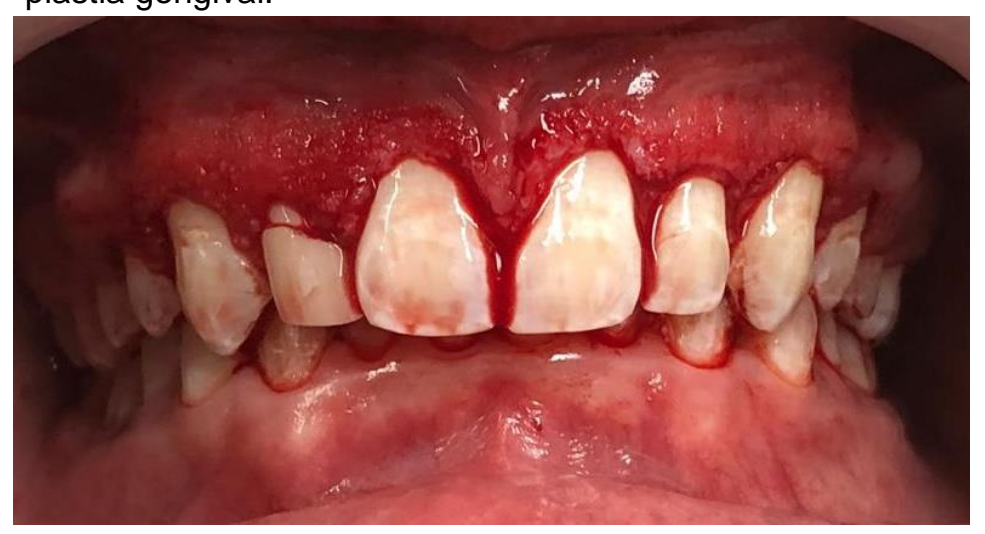

Fonte: Silva QP, et al., 2020.

Figura 3 - Pós-operatório de 30 dias.

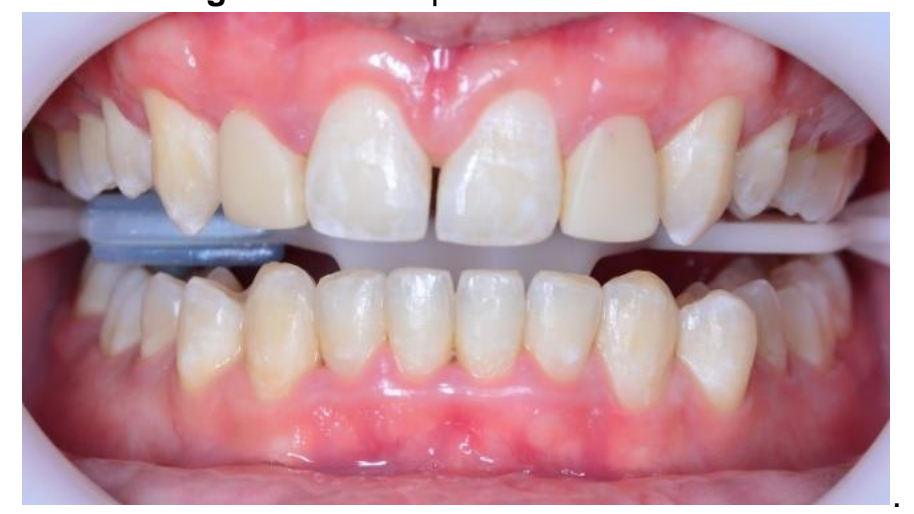

Fonte: Silva QP, et al., 2020.

Pós-operatório de 30 dias logo após foi iniciada a incisão com cabo de bisturi №3 (Golgran®) e lâmina de bisturi $N^{\circ} 15 \mathrm{c}$, a incisão foi feita em elipse acompanhando o contorno do freio nos dois lados até a região de linha mucogengival, com as incisões convergindo nessa região e resultando na remoção do fragmento do freio labial (Figura 4A). Após isso foi realizado o rompimento do plano intermediário do freio com o auxílio da lâmina de bisturi, para que não houvesse uma reinserção do freio na mesma posição.

Vale salientar também que se optou por não englobar a área de papila de forma direta na intervenção, visto a dificuldade de regeneração dos tecidos dessa área em caso de dano, assim, após a remoção do fragmento do freio labial, uma incisão intrasulcular foi realizada na papila entre os elementos 11 e 21 , seguida de descolamento mucoperiosteal com curta de Molt $N^{\circ} 2$ (Millenium Golgran ${ }^{\circledR}$ ) e com auxílio da lâmina de bisturi foi removido o plano intermediário que poderia estar presente nesta área (Figura 4A). 
Figura 4 - (A) Aspecto cirúrgico após remoção do fragmento de freio labial (B) Aspecto cirúrgico após descolamento mucoperiosteal da região de papila interdental.

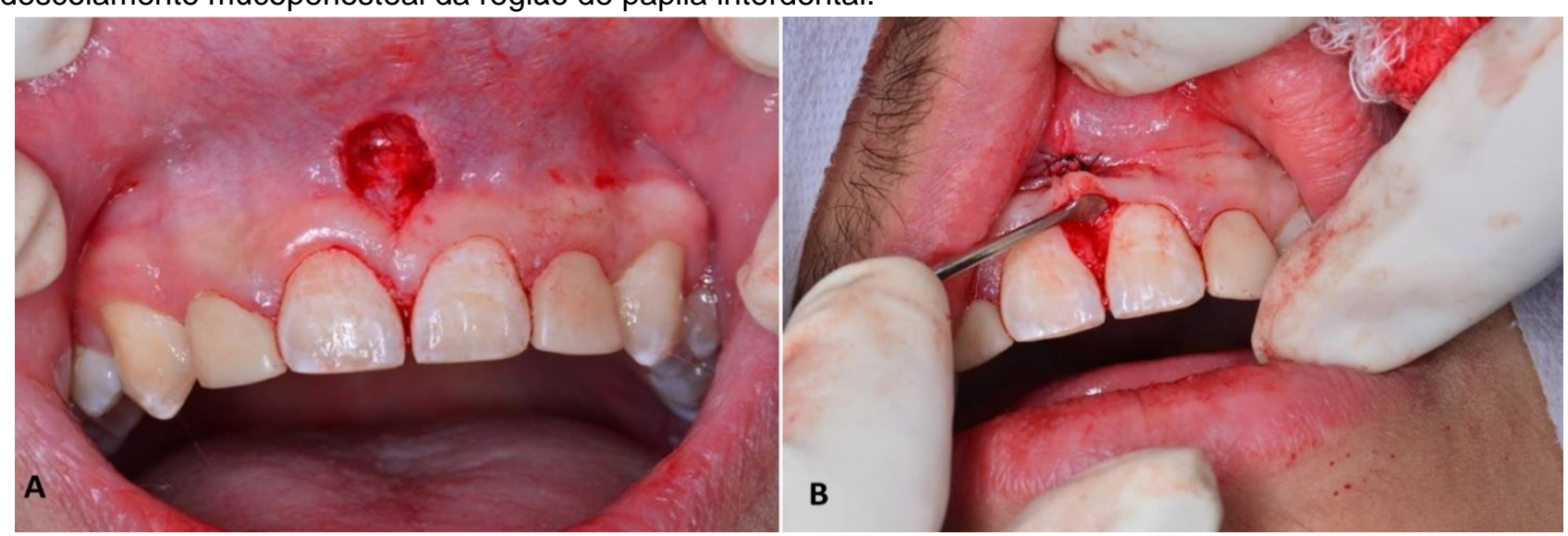

Fonte: Silva et al., 2020.

Após isso foi realizada a síntese da ferida cirúrgica com auxílio de um porta agulha Castroviejo $11 \mathrm{~cm}$ (Quinelato ${ }^{\circledR}$ ) e fio de sutura de Nylon 5.0 (Procare ${ }^{\circledR}$ ) sutura em pontos simples na região mais apical e sutura em colchoeiro vertical na região de papilla (Figura 5B). Um retorno foi agendado para 7 dias após a intervenção cirúrgica para remoção de sutura e avaliação do paciente, decorrido esse tempo, o mesmo não relatou nenhuma intercorrência e apresentou uma boa cicatrização (Figura 5A).

Figura 5 - (A) Pós-operatório imediato com ferida cirúrgica após sutura. (B) Aspecto clínico pós-operatório de 7 dias.

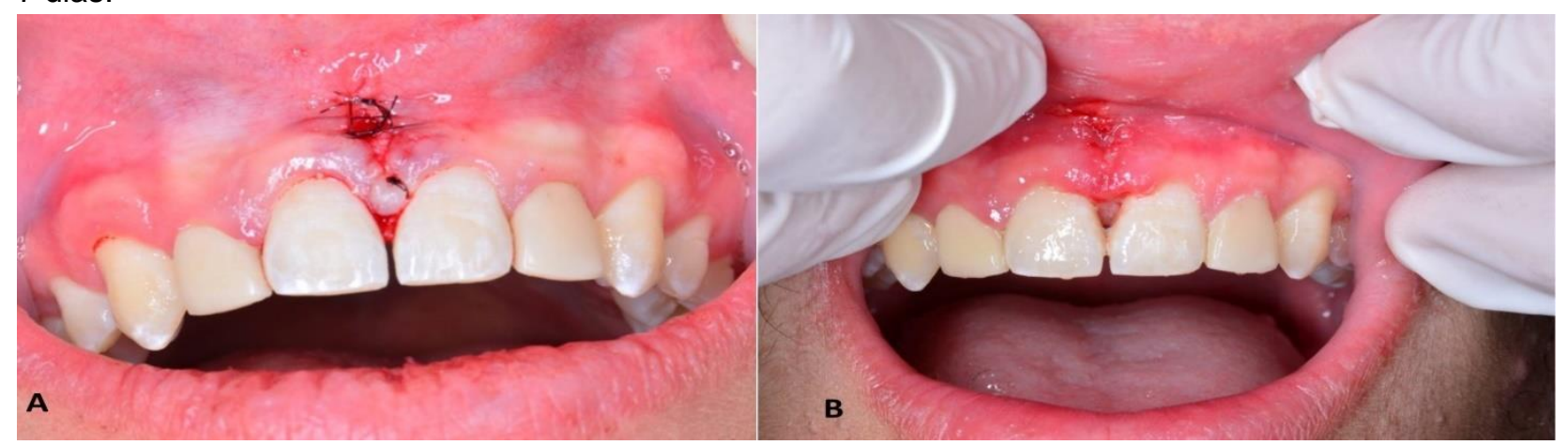

Fonte: Silva et al., 2020.

\section{DISCUSSÃO}

O presente relato descreveu um caso clínico de EPA do tipo I e subtipo A e o tratamento realizado para sua correção, bem como também descreveu o reposicionamento do freio labial devido sua inserção após a correção do sorriso gengival ter ficado baixa, comprometendo a estética do paciente.

No caso em questão, o paciente apresentava sorriso expandido e com linha alta, deixando claramente visível o tamanho reduzido das coroas clínicas desde o elemento 14 até $\circ 24$, não apresentando alterações de cor nos elementos e gengiva, e alteração de forma apenas no elemento 12 onde observou-se anatomia conóide, também notou-se uma espessura de tecido ceratinizado em excesso na região de gengiva inserida, o que comprometia sua estética. O mesmo não relatou dificuldade em higienizar a cavidade oral e não apresentava sangramento gengival a sondagem em $10 \%$ ou mais de todas as áreas analisadas.

No que se refere ao tratamento do sorriso gengival, a literatura é escassa e não muito fundamentada, sem dados de análise estatística que possam ser suportados. Os relatos de caso clínico frequentemente narram o uso da cirurgia periodontal sem deixar claras as diferenças entre a gengivectomia e a cirurgia ressectiva óssea (CAIRO F, et al., 2012; LOBO NS, et al., 2019). 
O tipo de cirurgia plástica periodontal a ser adotado está na dependência de vários fatores como: posição dos tecidos supracrestais, ou seja, se existe pelo menos 2,5 a $3 \mathrm{~mm}$ entre a JCE e a crista óssea e também se após a cirurgia haverá uma quantidade adequada de mucosa ceratinizada residual.

Caso encontremos esses fatores a gengivectomia sem confecção de retalho e osteotomia está indicada, caso não estejam presentes, a confecção ou não (no caso da intervenção minimamente invasiva) de retalho e osteotomia se fazem necessárias (ROCHA EF, et al., 2019).

No presente caso clínico houve acompanhamento de 3 meses devido a realização das duas intervenções cirúrgicas, e durante todo esse tempo o resultado e aparência clínica se mantiveram satisfatórios. Corroborando com o resultado obtido através da realização de gengivectomia com gengivoplastia e seu sucesso clínico, o estudo de Levine RA e Mcguirre M (1997), também relatou o sucesso do tratamento de dois casos clínicos de EPA do tipo I e subtipo A através apenas da gengivectomia com gengivoplastia sem confecção de retalho e osteotomia.

Outro fator que pode comprometer a estética do paciente é a posição do freio labial, onde o periodontista ou cirurgião pode escolher dentre muitas técnicas a que melhor se encaixa para correção desse problema. $\mathrm{O}$ comprometimento estético se dá pela inserção baixa do freio, que pode ser devido a características congênitas podendo resultar na presença de diastemas, ou pode surgir após intervenções periodontais como a gengivectomia que altera a posição da margem gengival e consequentemente a altura de inserção do freio, o que pode interferir na harmonia do sorriso (BRUDER C, et al., 2015; GOMES JT, et al., 2019).

A literatura relata várias técnicas para remoção do freio, como a da "borda triangular" e a em forma de "V" (MORSELLI P, et al., 1999; EDWARDS JG, 1977). Já a técnica utilizada no presente caso, foi semelhante a proposta por Chelotti no estudo de Bruder et al (2015), porém seguindo as incisões em elipse tangenciais nas bordas laterais do freio. A técnica de Chelloti é frequentemente utilizada na odontopediatria para reposicionamento de freio teto labial persistente e apresenta bons resultados (SILVA HL, et al., 2018).

Como resultado final no caso, foi observado a correção do contorno gengival com consequente obtenção da harmonia estética do sorriso, confirmando a eficácia do tratamento proposto com intervenção cirúrgica através de uma gengivectomia em bisel externo e plastia gengival em toda área compreendida entre os elementos 14 ao 24, validando, assim como Alvarengua DV, et al. (2018), a importância do correto diagnóstico e planejamento cirúrgico prévio levando em consideração todos os fatores anatômicos, funcionais e psicológicos do paciente.

A técnica de gengivectomia em bisel externo e plastia gengival no tratamento da EPA pode se mostrar uma ótima alternativa nos casos de sorriso gengival ocasionado por erupção passiva alterada, podendo assim, devolver a harmonia estética do sorriso ao paciente.

\section{REFERÊNCIAS}

1. ALVARENGUA DV, et al. Inter-relação na periodontia/dentística na correção de sorriso gengival: relato de caso clínico. Periodontia. 2018; 28(2): 53-59.

2. BRUDER $\mathrm{C}$, et al. Frenectomia labial pela técnica de reposicionamento cirúrgico proposta por Chelotti, Revista Metodista, 2015; 23 (45): 45-46.

3. CAIRO F, et al. Periodontal Plastic Surgery to Improve Aesthetics in Patients with Altered Passive Eruption /Gummy Smile: A Case Series Study. International Journal of Dentistry, 2012; 2012: 1-6.

4. CAVALCANTE JÁ, et al. Diagnóstico e tratamento cirúrgico do freio teto labial persistente em pacientes no período intertransitório da dentição mista-relato de caso. Rev Inst Ciênc Saúde. 2009; 27(3): 290-294.

5. CRISTÓVAM AVS, et al. Correção de contorno gengival pelas técnicas de gengivectomia convencional e minimamente invasiva. Archives Of Health Investigation, 2020; 8 (10): 606-612.

6. DUTRA MB, et al. Influência da exposição gengival na estética do sorriso. Dental Press J Orthod. 2011; 16(5): 111118.

7. EDWARDS JG. The diastema, the frenum, the frenectomy: a clinical study. Am J Orthod. 1977; 71 (5): $489-508$.

8. GOMES JT, et al. Gengivectomias em terapias gengivais: Relato de caso. Jornal Internacional de Odontologia Clínica, $2019 ; 12(1): 83-90$.

9. LEVINE RA, MCGUIRE M. The diagnosis and treatment of the gummy smile. Compendium, 1997; 18: $757-764$. 
10. LOBO NS, et al. Cirurgia periodontal de aumento de coroa clínica estética sem a elevação do retalho (flapless): relato de caso clínico. Arquivo Brasileiro De Odontologia, 2019; 13(1): 118-123.

11. MORSELLI P, et al. Frenuloplasty by means of a triangular flap. Oral Surg Oral Med Oral Pathol Oral Radiol Endod. 1999; 87 (2):142-144.

12. ROCHA EF, et al. Harmonização do sorriso através da cirurgia plástica periodontal: um relato de caso. Revista Eletrônica Acervo Saúde, 2019; 26: e780.

13. SEIXAS MR, et al. Checklist dos aspectos a serem considerados no diagnóstico e tratamento do sorriso gengival. Dental Press J Orthod. 2011;16(2):131-57.

14. SILVA HL, et al. Frenectomia: revisão de conceitos e técnicas cirúrgicas. Salusvita, 2018; 37 (1): 139-150.

15. VIEIRA PR, et al. Técnica de frenotomia para correção de freio labial superior em odontopediatria. Revista uningá review, 2014; 19 (3): 41-43. 